\title{
Evaluation of Service Life of Polystyrene in Tropical Marine Environment by Principal Component Analysis
}

\author{
Dongdong Song, Jin Gao, Lin Lu, and Xiaogang Li \\ Corrosion and Protection Center, University of Science and Technology Beijing, Beijing 100083, China \\ Correspondence should be addressed to Jin Gao; gaojin@ustb.edu.cn
}

Received 4 January 2015; Revised 23 March 2015; Accepted 2 April 2015

Academic Editor: Peter Chang

Copyright (C) 2015 Dongdong Song et al. This is an open access article distributed under the Creative Commons Attribution License, which permits unrestricted use, distribution, and reproduction in any medium, provided the original work is properly cited.

\begin{abstract}
To predict the service life of polystyrene (PS) under an aggressive environment, the nondimensional expression $Z$ was established from a data set of multiple properties of PS by principal component analysis (PCA). In this study, PS specimens were exposed to the tropical environment on Xisha Islands in China for two years. Chromatic aberration, gloss, tensile strength, elongation at break, flexural strength, and impact strength were tested to evaluate the aging behavior of PS. Based on different needs of industries, each of the multiple properties could be used to evaluate the service life of PS. However, selecting a single performance variation will inevitably hide some information about the entire aging process. Therefore, finding a comprehensive measure representing the overall aging performance of PS can be highly significant. Herein, PCA was applied to obtain a specific property $(Z)$ which can represent all properties of PS. $Z$ of PS degradation showed a slight decrease for the initial two months of exposure after which it increased rapidly in the next eight months. Subsequently, a slower increase of $Z$ value was observed. From the three different stages shown as $Z$ value increases, three stages have been identified for PS service life.
\end{abstract}

\section{Introduction}

In the last four decades, the use of polymeric materials has rapidly increased but it is well established that these materials are susceptible to rapid photodegradation when exposed to natural weathering [1-4]. This is a serious issue, with economic and environmental implications. Therefore, a large effort is focused on understanding the relationship between the degradation kinetics and weathering environments [58]. The influences of ultraviolet radiation [9], oxygen [10], chemical composition [11], and degradation products [12, 13] to the aging mechanisms have been demonstrated by previous research, providing a good basis for the prediction of service lives of polymeric materials. Many laboratory accelerated weathering tests [14-17] were also performed.

The ability to accurately predict the long-term performance of polymers is essential both for the polymer industry and for those industries that use those polymers on their products. There are two questions that are usually asked. First, natural environment is different from the testing environment in the accelerated studies. Temperature, rainfall, and relative humidity are very important environmental factors that can be reproduced in a laboratory environment. Natural weathering is the most reliable exposure method $[18,19]$. To the best of our knowledge, little has been reported on the aging behaviors of PS in the tropical marine environment after a short time exposure, for example, $<10$ years. Xisha archipelago of China has a typical tropical marine climate, characterized with high temperature, high humidity, and long daylight. Second, with which property will we define the failure? For polymer, appearance variation and mechanical loss are the main concerns. But, the variation of each property is usually very different. Thus, it is meaningful to find a single parameter as the criterion for polymer failures.

In this study, degradation of PS was investigated in Xisha atmospheric corrosion site in a 2-year exposure program and the results obtained were analyzed and discussed as a function of the exposure time. To evaluate the aging behavior, the analyses on the appearance and mechanical performance of PS are often suggested. However, the trend for each property may be different and selecting a single performance change will inevitably hide some information about the entire 
aging process. Therefore, finding a comprehensive measure of representing the overall aging performance of PS becomes critical.

Principal component analysis (PCA) is a multivariate statistical method that can be used to reduce data dimensionality and evaluate multivariate objectively. A number of possibly correlated variables could be transformed into a smaller number of uncorrelated variables by PCA. And the smaller number of uncorrelated variables is called principal components. It can be considered as a process for extracting hidden, simplified structures within the data in a way which best explains the variance in the data [20]. Zhao et al. evaluated the degradation behavior of EPDM upon exposure to an artificial weathering environment produced by xenon arc lamp using PCA [21]. In separate studies, the authors have used PCA to evaluate the aging behaviors of PE and PP in the natural environment of Xisha Islands [22, 23].

To study the aging characteristics of PS, changes in appearance and physical properties were usually monitored. For example, the increase of gloss loss was used to indicate the aggravation of the aging process of PS. However, each single property can only reflect one side of the aging behavior. If the effects of all properties are considered, the evaluation might be contradictory sometimes. Such dilemma can be solved by using a PCA method which could reduce the redundancy in these properties and extract a smaller number of principal components (artificial variables). After that, a comprehensive expression can be built up by the extracted components to represent the majority of multiple original properties. Herein, the aging of PS in Xisha atmospheric environment was evaluated using a PCA method.

\section{Experimental}

2.1. Exposure Test. Natural exposure tests were performed under the tropical environment in Xisha Islands with an average temperature of $28^{\circ} \mathrm{C}$, an average humidity of $85 \%$, and an average sunlight duration of $150 \sim 280 \mathrm{~h}$ per month. PS samples (supplied by Chenguang Research Institute of Chemical Industry, China) were exposed to the natural environment on a shelf with an angle of $45^{\circ}$ to the horizontal according to ISO 877:1994 for 2 years. The specific exposure duration was from January 2008 to January 2010 and the specimens were retrieved after $1,2,3,6,9,12,15,18$, and 24 months.

\subsection{Measurements}

\subsubsection{Appearance}

(a) The Macroscopic Morphology. PS morphologies were compared after outdoor exposure of various cycles.

(b) Chromatic Aberration Measurement. Chromatic aberration was measured with a spectrophotometer (GretagMacbeth COLOREYE XTH, USA). The change of color $\Delta E$ was calculated by

$$
\Delta E=\sqrt{\Delta L^{2}+\Delta a^{2}+\Delta b^{2}}
$$

where $\Delta L$ represents the brightness relationship between light and dark; $\Delta a$ represents the relationship between green and red, and $\Delta b$ represents the relationship between blue and yellow. The symbol $\Delta$ implies the difference between the samples before and after aging.

(c) Gloss Measurement. Surface gloss was measured through a portable glossmeter (XGP, China) using a $60^{\circ}$ incidence angle.

(d) Hardness. The indentation hardness (Shore D) of the exposed side of the plate samples was determined via a pocket hardness meter (type TH210, China) according to ISO 868:2003. The average value was taken from at least five different locations.

\subsubsection{Mechanical Properties}

(a) Tensile Strength. The tensile specimens were tested according to ISO 527:2012 at room temperature $\left(23^{\circ} \mathrm{C}\right)$ with a crosshead speed of $20 \mathrm{~mm} / \mathrm{min}$ using a computer-controlled universal testing machine (type WDS, China). $\sigma$ can be calculated by

$$
\sigma=\frac{P}{b d}
$$

in which $\sigma$ is tensile strength in $\mathrm{MPa}, P$ is maximum load, and $b$ and $d$ are the width and thickness of sample in $\mathrm{mm}$, respectively.

The elongation at break $(\varepsilon, \%)$ can be calculated by

$$
\varepsilon=\frac{G-G_{0}}{G_{0}} \times 100
$$

where $G_{0}$ and $G$ are the elongation length in $\mathrm{mm}$ before and after test, respectively.

(b) Flexural Strength. The exposure side of sample was pressed with the preload of $5 \mathrm{~N}$ and the speed of $5 \mathrm{~mm} / \mathrm{min}$ using a computer-controlled universal testing machine (type WDS, China). The flexural strength $(\sigma, \mathrm{MPa})$ was obtained via

$$
\sigma=\frac{3 P \cdot L}{2 b \cdot h^{2}}
$$

where $P$ is the compressive load in $\mathrm{N} ; L$ is the cross length in $\mathrm{mm} ; b$ and $h$ are the width and the thickness of sample in $\mathrm{mm}$, respectively.

(c) Impact Strength. According to the standard ISO 179-2010, electronic impact testing machine (XJJD China) was used for impact test by $5 \mathrm{~J}$ pendulum at room temperature. Impact strength is calculated as follows:

$$
\alpha=\frac{W}{b \cdot h} \times 10^{3}
$$

in which $\alpha$ is the impact strength in $\mathrm{kJ} / \mathrm{m}^{2} ; W$ is the amended energy of the sample's destruction in J. $b$ and $h$ are the width and the thickness of the sample, respectively. 
2.3. Evaluation Method. The idea of PCA is to reduce dimensionality of variables. A smaller number of variables called principal components are linear combinations of original variables, which are orthogonal to each other. The number of principal components is chosen based on the variance they are responsible for. PCA was conducted by using SPSS software (version 11.5, SPSS Inc., Chicago, USA). The original variables used to characterize the degradation behavior of PS before and after exposure to natural environment are yellow index $(X 1)$, color aberration $(X 2)$, the change gloss $(X 3)$, the change of impact strength $(X 4)$, the change of tensile strength $(X 5)$, the change of elongation at break (X6), and the change of flexural strength $(X 7)$.

\section{Results and Discussion}

3.1. Appearance. In Figure 1, the transparency of the original sample is relatively good, as the grid below the sample can be clearly seen. With extended duration of outdoor exposure, the transparency of the sample is gradually reduced and the color of sample changes to yellow after 6 months. The reason for such yellowing has been attributed to the formation of conjugated double bonds along the polystyrene backbone and also the formation of fulvene derivatives by photoisomerization of the phenyl groups in PS [24].

The grid below sample became very fuzzy after 12 months of exposure and was basically concealed after 24 months. Macroscopic cracks cannot be observed on the surface of the sample after 24 months, indicating that aging is not serious.

The change in sample's chemical structure resulting from photoaging decreases the reflection and absorption of light, which can be quantified as the loss of gloss. As shown in Figure 2, the gloss of the sample drops slowly from 0 to 4 months of exposure and more rapidly from 4 to 6 months. During 6 to 9 months, the gloss is relatively unchanged. After that, the gloss of the sample drops again. Based on the gloss variation, the aging process of PS can be divided into four stages including a slow changing prophase before 4 months of exposure, a rapid changing prophase from 4 to 6 months of exposure, a relatively stable anaphase from 6 to 9 months of exposure, and a slow changing prophase from 10 to 24 months.

With longer exposure, the color of sample gradually becomes darker. And this is consistent with the measurement of chromatic aberration (Figure 2). Chromatic aberration increases rapidly in the first 6 months, followed by a slower increase to the maximum value at 24 months. The lightinduced aging process starts from the surface of PS and chromatic aberration is a reflection of sample's surface information. Thus, a sharp change of chromatic aberration can be observed at a very early stage of the exposure. As exposure continues, the aging process gradually penetrates from the surface to the interior of the sample, leading to a decrease in the variation of chromatic aberration. This is represented by the fact that the change of chromatic aberration is slower than that of gloss in the later stage.

Unlike gloss, the aging process of PS can be divided into two steps by analyzing the result of chromatic aberration. The prophase is the rapid change step before 6 months of exposure and the metaphase is the steady change step from 7 to 24 months of exposure.

Figure 3 shows that hardness remained unaffected by the harsh environment during the entire exposure time. It means that hardness cannot reflect the aging behavior of PS.

3.2. Mechanical Properties. Mechanical testing is important for revealing the aging behavior of polymers. Tensile strength, elongation at break, bending strength, and impact strength are the main indicators of the aging resistance of PS materials.

3.2.1. Tension Test. The tensile strength of samples after different exposure duration was tested by the universal testing machine. In order to show the trend of changes in tensile properties more clearly, retention rate was plotted instead of tensile strength.

In Figure 4, the tensile strength exhibits an obvious increase (20\%) during the first 2 months of exposure. Then, it decreased markedly, showing only $50 \%$ of the initial value after 6 months. The declined tensile strength for these samples could be attributed to the oxidation of the polymer by chain scissions under UV exposure. According to the variation in tensile strength, the aging process of PS can be categorized into three steps, that is, no degradation step (before 2 months of exposure), rapid changing step (from 3 to 6 months of exposure), and stable step (from 7 to 24 months of exposure).

For the values of elongation at break, the trend is the same as tensile strength. But, it decreases more rapidly. This implies that the toughness of PS sample is more sensitive to the exposure than the strength. The change of the elongation also shows that the aging process of PS has three steps.

3.2.2. Flexure and Impact Test. Flexural strength and impact strength are also important properties indicating the aging behaviors of polymers. Different to the trend recorded for the tensile strength, flexural strength decreases gradually throughout the entire exposure (Figure 5). This can be attributed to the increased brittleness and stiffness of PS caused by photooxidation. The aging process of PS can be divided into two steps based on the change in flexural strength, that is, a rapid changing step (before 18 months of exposure) and a steady changing step (from 18 to 24 months of exposure).

Unlike the flexural strength, the impact strength fluctuates at the first 6 months of exposure. After 6 months, it drops rapidly. Apparently, the impact strength is more sensitive to the exposure than the flexural strength. This might be attributed to the formation of deep surface cracks which causes a ductile to brittle transition of the polymer, losing its original toughness.

Similar to the result of flexural strength, the variation of tensile impact strength shows that the aging process of PS has three steps. Zero-six months of exposure is the fluctuation step; 6-12 months of exposure is the rapid changing step; $12-$ 24 months of exposure is the stable step. 


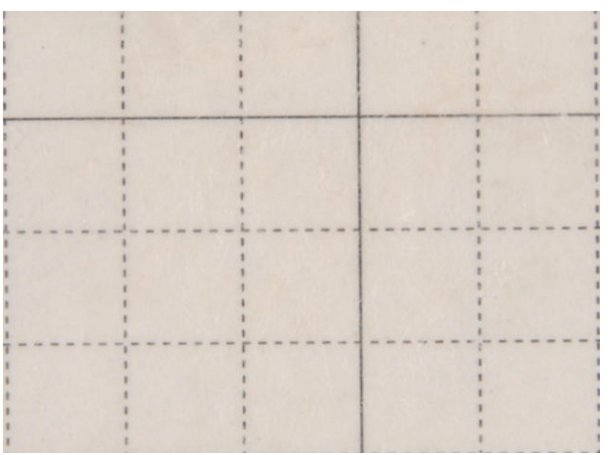

(a)

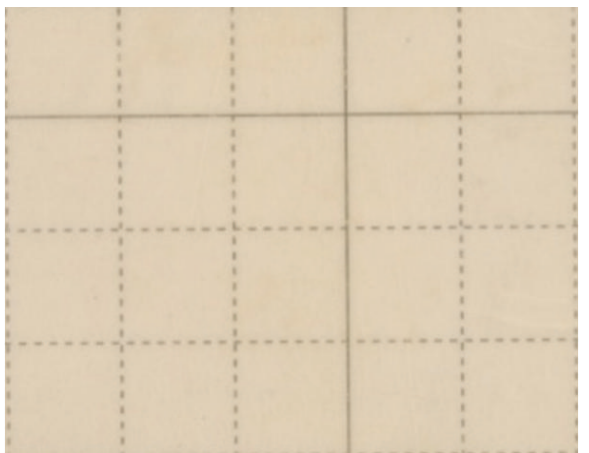

(c)

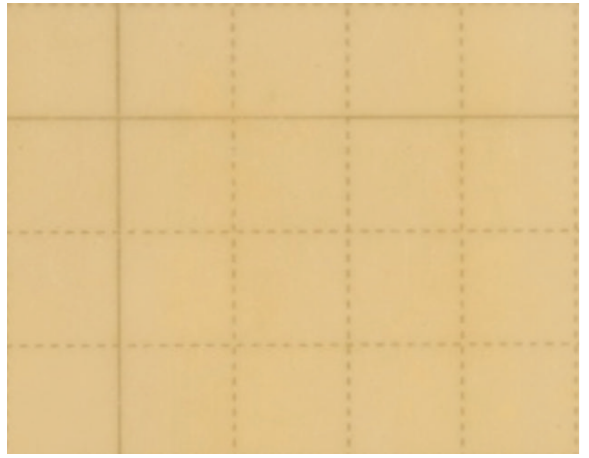

(e)

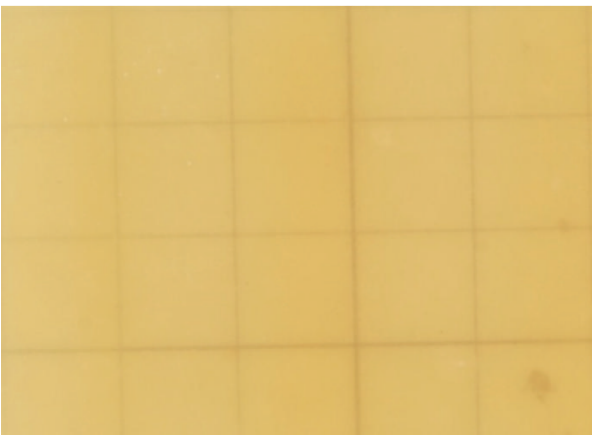

(g)

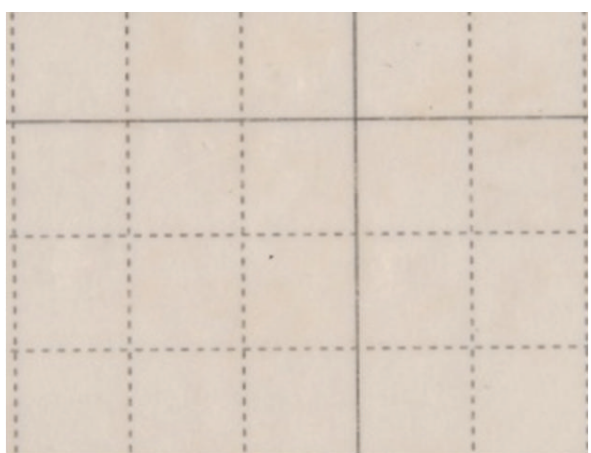

(b)

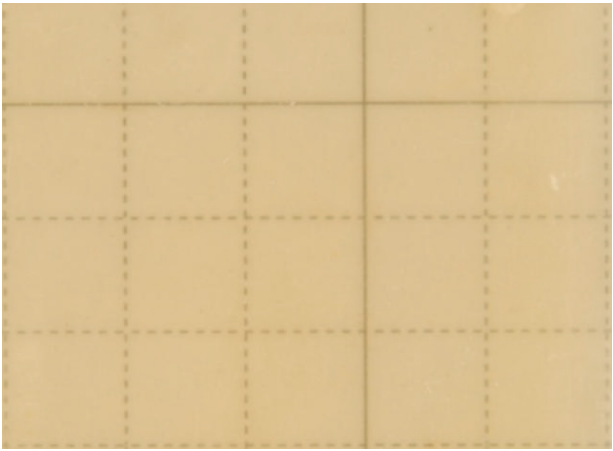

(d)

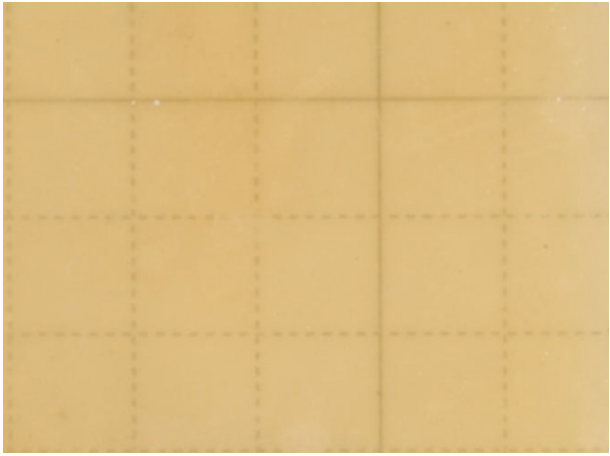

(f)

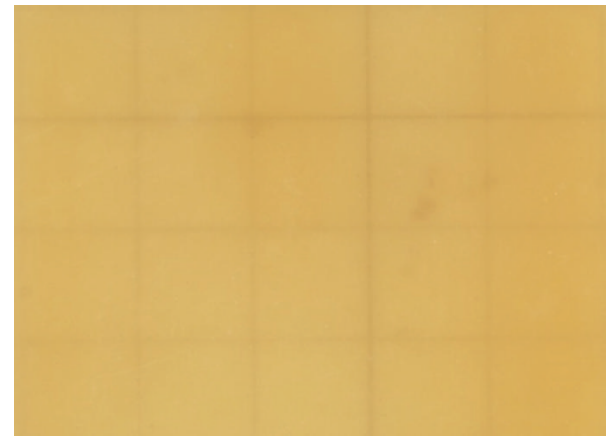

(h)

FIGURE 1: Surface morphology of PS exposed in Xisha Island: (a) original sample; (b) 1-month exposure; (c) 3-month exposure; (d) 6-month exposure; (e) 9-month exposure; (f) 12-month exposure; (g) 18-month exposure; (h) 24-month exposure. 


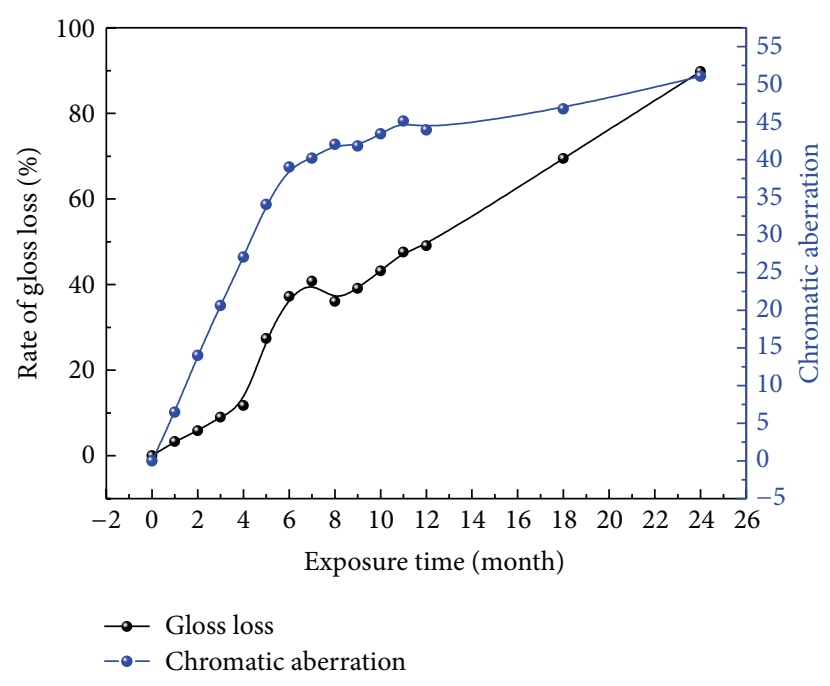

FIGURE 2: Change of rate of gloss loss and chromatic aberration of PS with extended exposure in the environment of Xisha Islands.

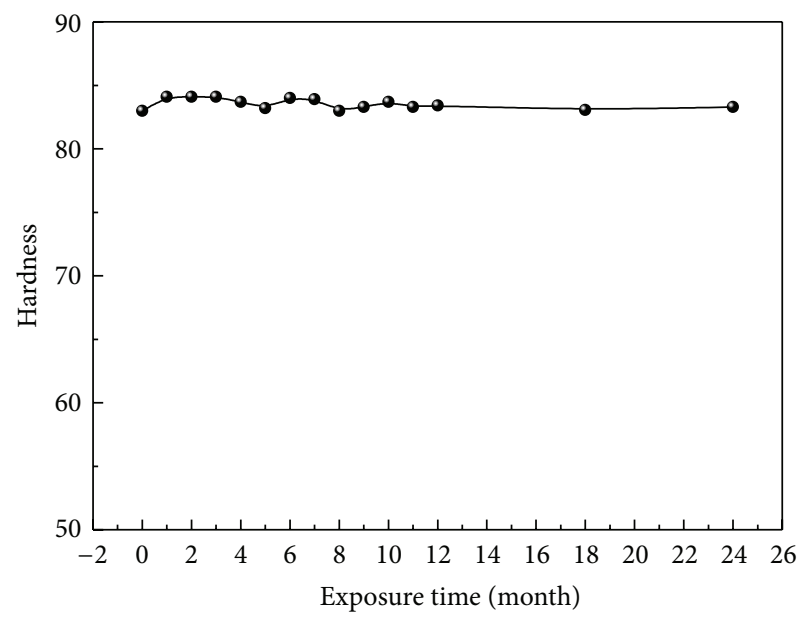

FIGURE 3: Relationship between exposure time and the hardness of PS under the environment of Xisha Island.

3.3. Evaluation. The degradation process of PS can be reflected by variations in its aesthetic properties such as chromatic aberration and gloss and mechanical properties such as tensile strength, elongation at break, flexural strength, and impact strength. Unfortunately, the degradation behavior evaluated by each individual property of PS might be different. Therefore, it is quite difficult to find a specific property to represent all others, to contain all the effective information and define the failure of PS.

The idea of PCA is to reduce dimensionality of variables. A smaller number of variables called principal components are linear combinations of original variables, which are orthogonal to each other. The number of principal components is chosen based on the variance they are responsible for. In our study, the original variables used to characterize the degradation behavior of PS before and after exposure to natural environment are yellow index $(X 1)$, color aberration $(X 2)$,

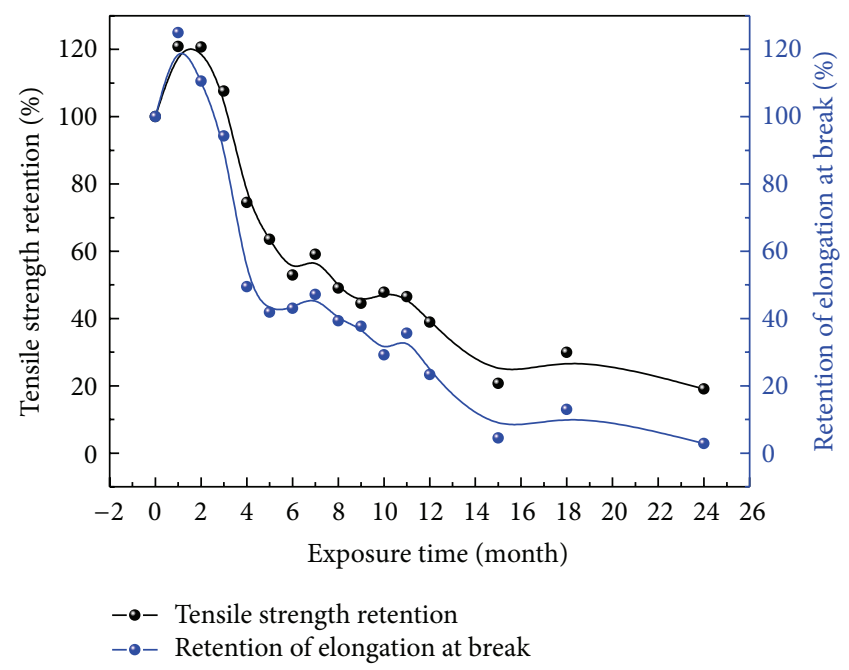

FIgURE 4: Change of the retention of tensile strength and that of elongation at break of PS with extended exposure time under the environment of Xisha Islands.

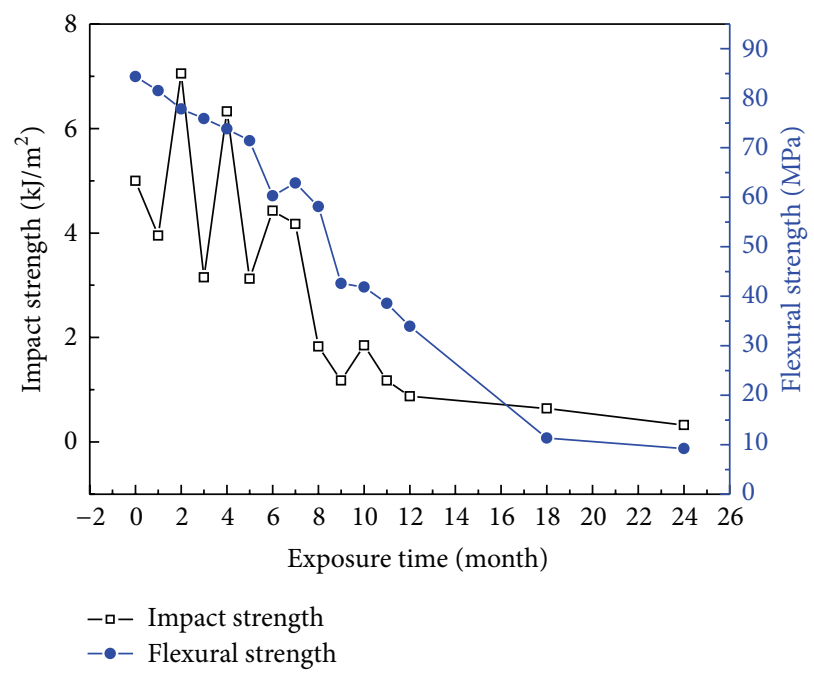

FIGURE 5: Change of the flexural strength and impact strength of PS with extended exposure time in the environment of Xisha Islands.

the change of gloss $(X 3)$, the change of impact strength $(X 4)$, the change of tensile strength $(X 5)$, the change of elongation at break (X6), and the change of flexural strength (X7). PCA was carried out by using SPSS software (version 11.5, SPSS Inc., Chicago, USA). Hardness was not utilized and because of that it did not change obviously for the total exposure.

The results from PCA show that when the number of principal components is two, the total variance is $95.224 \%$, which means that two principal components contain $95.224 \%$ of the total information reflected by the seven degradation parameters. The two principal components $Y 1$ and $Y 2$ can be expressed as

$$
\begin{aligned}
Y 1= & 0.392251 X 1+0.382245 X 2+0.384246 X 3 \\
& +0.335815 X 4+0.39065 X 5+0.383846 X 6 \\
& +0.373439 X 7
\end{aligned}
$$




$$
\begin{aligned}
Y 2= & -0.2104 X 1-0.30408 X 2+0.110573 X 3 \\
& +0.747904 X 4-0.21807 X 5-0.35629 X 6 \\
& +0.34247 X 7 \\
Z= & \frac{\lambda_{1}}{\sum_{i=1}^{7} \lambda_{i}} Y 1+\frac{\lambda_{2}}{\sum_{i=1}^{7} \lambda_{i}} Y 2 \\
= & 0.89166 Y 1+0.06058 Y 2 .
\end{aligned}
$$

Figure 6 illustrates the loading plot of principal components. It can be seen that lines $X 1$ and $X 5$ are almost concurrent, suggesting that the yellow index is strongly associated with tensile strength. Line $X 2$ is close to line $X 6$, which also indicates that the color aberration is highly correlated with the elongation at break.

The first principal component reflects the effects of yellow index $(X 1)$, color aberration $(X 2)$, gloss $(X 3)$, tensile strength $(X 5)$, and elongation at break $(X 6)$, all of which are positively correlated. The second principal component reflects the effect of impact strength that is positively correlated. From the above analysis it can be inferred that principal component 1 represents comprehensive property and principal component 2 represents mechanical properties. And the principal components can define the failure of PS by customers.

PCA method was introduced to extract principal components from the aging properties by establishing a comprehensive expression. Combined evaluating parameters $(Z)$ are the principal component of all aging properties. Variation of $Z$ can be representative for the change of specimen and can predict the service lifetimes of polymers. It has been successfully used to evaluate the aging behavior of EPDM [21], MDPE [22], and PP [23].

The variation of $Z$ versus exposure time is presented in Figure 7. During the first two months, the combined evaluation parameter $Z$ decreases a little. It is the early stage of its service life. From three to five months of exposure, $Z$ values show the most rapid growth. This is defined as the middle stage of its service life. After that, $Z$ increases by a slower rate till 24 months, which marks the end of the service life of PS.

\section{Conclusions}

In this study, PS samples were exposed in natural environment on Xisha Islands for 2 years and their appearance and mechanical properties were analyzed at different exposure time. All properties showed that PS had gradually degraded but the service life of PS determined by each property may be different. Thus, PCA was used as an effective method to extract relevant information from seven different characteristic properties of PS and produce a single parameter representative of the overall aging behavior. During the twoyear exposure, the combined evaluating parameter $Z$ showed slight declines in the first two months (early stage of service life), after which it increased speedily (middle stage) and subsequently slowed down (end stage).

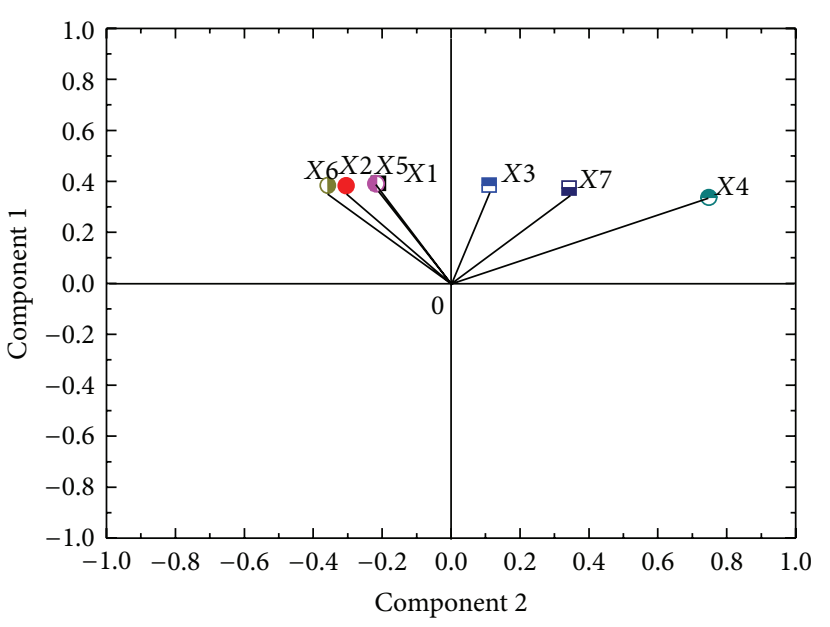

FIGURE 6: Loading plot of principal components.

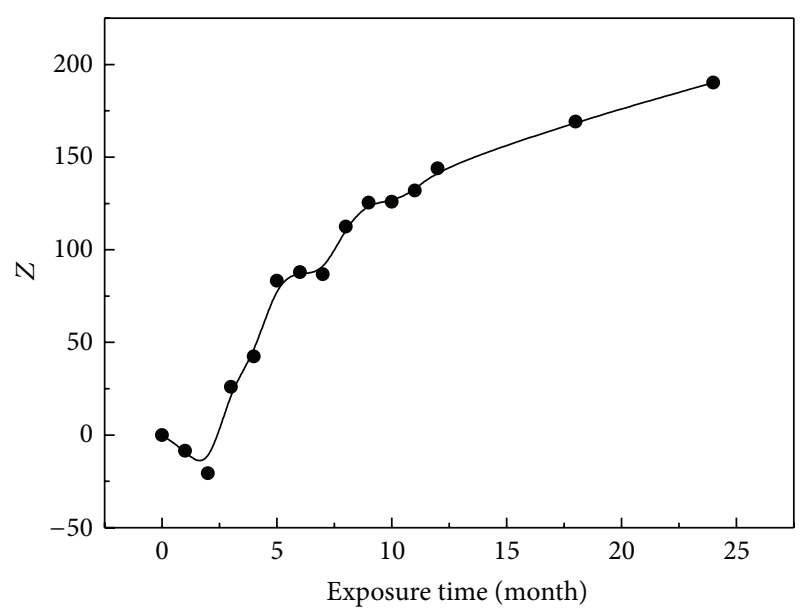

FIGURE 7: Variation of combined evaluating parameters on exposure time.

\section{Conflict of Interests}

The authors declare that there is no conflict of interests regarding the publication of this paper.

\section{Acknowledgments}

The authors wish to acknowledge the financial support of the National Natural Science Foundation of China (no. 51133009) and National Scientific Data Sharing and Service Network on Material Environmental Corrosion (Aging).

\section{References}

[1] F. A. Bottino, A. R. Cinquegrani, G. di Pasquale, L. Leonardi, and A. Pollicino, "Chemical modifications, mechanical properties and surface photo-oxidation of films of polystyrene (PS)," Polymer Testing, vol. 23, no. 4, pp. 405-411, 2004.

[2] A. Torikai, T. Kobatake, F. Okisaki, and H. Shuyama, "Photodegradation of polystyrene containing flame-retardants: 
wavelength sensitivity and efficiency of degradation," Polymer Degradation and Stability, vol. 50, no. 3, pp. 261-267, 1995.

[3] H. Kaczmarek, A. Kami\&apos; nska, M. \&apos; Swiątek, and S. Sanyal, "Photoinitiated degradation of polystyrene in the presence of low-molecular organic compounds," European Polymer Journal, vol. 36, no. 6, pp. 1167-1173, 2000.

[4] B. Mailhot, S. Morlat, and J.-L. Gardette, "Photooxidation of blends of polystyrene and poly(vinyl methyl ether): FTIR and AFM studies," Polymer, vol. 41, no. 6, pp. 1981-1988, 2000.

[5] Y. Azuma, H. Takeda, S. Watanabe, and H. Nakatani, "Outdoor and accelerated weathering tests for polypropylene and polypropylene/talc composites: a comparative study of their weathering behavior," Polymer Degradation and Stability, vol. 94, no. 12, pp. 2267-2274, 2009.

[6] T. Şahin, T. Sinmazçelik, and Ş. Şahin, "The effect of natural weathering on the mechanical, morphological and thermal properties of high impact polystyrene (HIPS)," Materials \& Design, vol. 28, no. 8, pp. 2303-2309, 2007.

[7] C. C. White, K. T. Tan, D. L. Hunston et al., "Laboratory accelerated and natural weathering of styrene-ethylene-butylenestyrene (SEBS) block copolymer," Polymer Degradation and Stability, vol. 96, no. 6, pp. 1104-1110, 2011.

[8] J. Jose, A. Nag, and G. B. Nando, "Environmental ageing studies of impact modified waste polypropylene," Iranian Polymer Journal, vol. 23, no. 8, pp. 619-636, 2014.

[9] N. A. Weir, P. Kutok, and K. Whiting, "Some aspects of the longwave photo-oxidation of polystyrenes," Polymer Degradation and Stability, vol. 24, no. 4, pp. 247-256, 1989.

[10] J. F. Rabek and B. Rånby, "Studies on the photooxidation mechanism of polymers. I. Photolysis and photooxidation of polystyrene," Journal of Polymer Science: Polymer Chemistry Edition, vol. 12, no. 2, pp. 273-294.

[11] J. F. Rabek and B. Rånby, "Studies on the photooxidation mechanism of polymers. II. The role of quinones as sensitizers in the photooxidative degradation of polystyrene," Journal of Polymer Science: Polymer Chemistry Edition, vol. 12, no. 2, pp. 295-306, 1974.

[12] N. A. Weir and T. H. Milkie, "The effects of degradation products on the rates of photo-reactions in polystyrene films," Polymer Photochemistry, vol. 7, no. 2, pp. 129-138, 1986.

[13] P. C. Lucas and R. S. Porter, "Autoinhibition in polystyrene photo-oxidation," Polymer Degradation and Stability, vol. 26, no. 3, pp. 203-208, 1989.

[14] T. Yuzawa, C. Watanabe, N. Nemoto, and H. Ohtani, "Rapid evaluation of photo, thermal and oxidative degradation of high impact polystyrene by a xenon lamp-based online ultraviolet irradiation-pyrolysis-GC/ MS system," Polymer Degradation and Stability, vol. 98, no. 2, pp. 671-676, 2013.

[15] F. P. la Mantia and M. Morreale, "Accelerated weathering of polypropylene/wood flour composites," Polymer Degradation and Stability, vol. 93, no. 7, pp. 1252-1258, 2008.

[16] B. P. Jelle and T.-N. Nilsen, "Comparison of accelerated climate ageing methods of polymer building materials by attenuated total reflectance Fourier transform infrared radiation spectroscopy," Construction and Building Materials, vol. 25, no. 4, pp. 2122-2132, 2011.

[17] J. W. Martin, T. Nguyen, E. Byrd, B. Dickens, and N. Embree, "Relating laboratory and outdoor exposures of acrylic melamine coatings. I. Cumulative damage model and laboratory exposure apparatus," Polymer Degradation and Stability, vol. 75, no. 1, pp. 193-210, 2002.
[18] D. A. Cocuzzi and G. R. Pilcher, “Ten-year exterior durability test results compared to various accelerated weathering devices: joint study between ASTM International and National Coil Coatings Association," Progress in Organic Coatings, vol. 76, no. 6, pp. 979-984, 2013.

[19] F. X. Perrin, C. Merlatti, E. Aragon, and A. Margaillan, "Degradation study of polymer coating: improvement in coating weatherability testing and coating failure prediction," Progress in Organic Coatings, vol. 64, no. 4, pp. 466-473, 2009.

[20] I. Jolliffe, Principal Component Analysis, Wiley, 2005.

[21] Q. Zhao, X. Li, J. Gao, and Z. Jia, "Degradation evaluation of ethylene-propylene-diene monomer (EPDM) rubber in artificial weathering environment by principal component analysis," Materials Letters, vol. 63, no. 1, pp. 116-117, 2009.

[22] L. Lu, X. Li, and J. Gao, "Evaluation of aging behavior of medium density polyethylene in natural environment by principal component analysis," Journal of Applied Polymer Science, vol. 125, no. 1, pp. 19-23, 2012.

[23] D. Song, J. Gao, X. Li, and L. Lu, "Evaluation of aging behavior of polypropylene in natural environment by principal component analysis," Polymer Testing, vol. 33, no. 1, pp. 131-137, 2014.

[24] G. Wypych, Handbook of Material Weathering, ChemTec Publishing, 2003. 

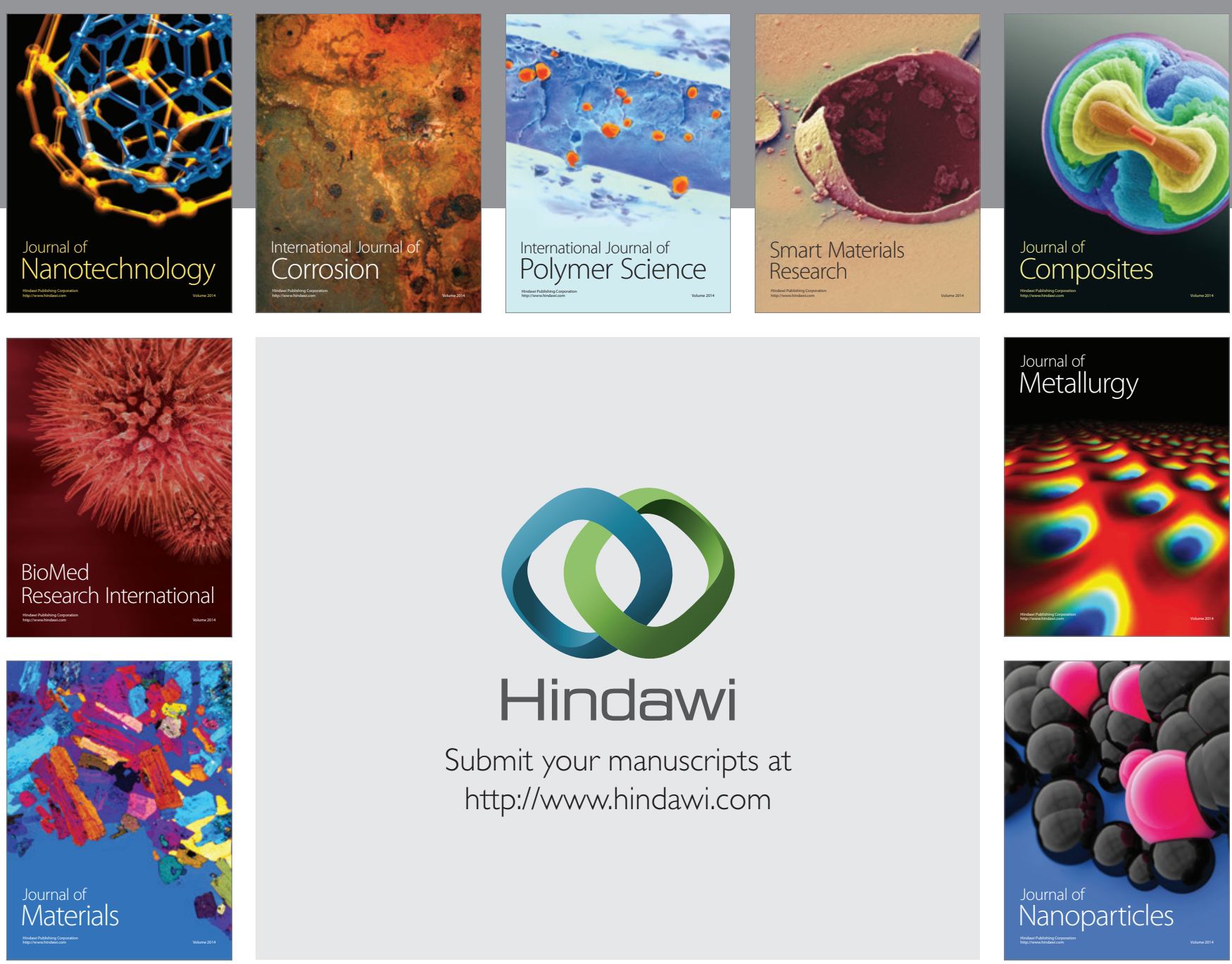

Submit your manuscripts at http://www.hindawi.com
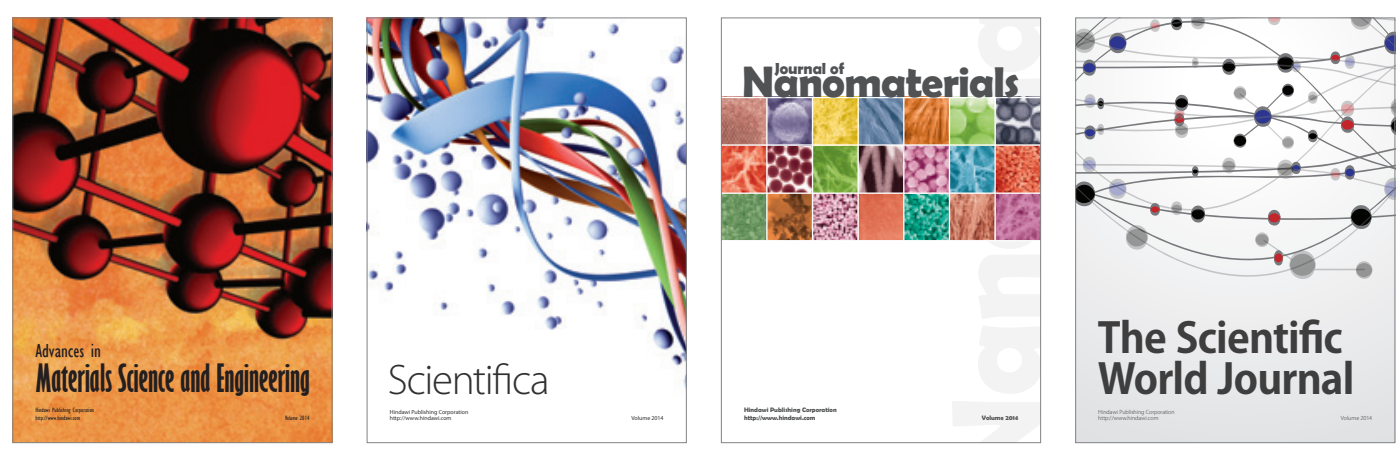

\section{The Scientific World Journal}
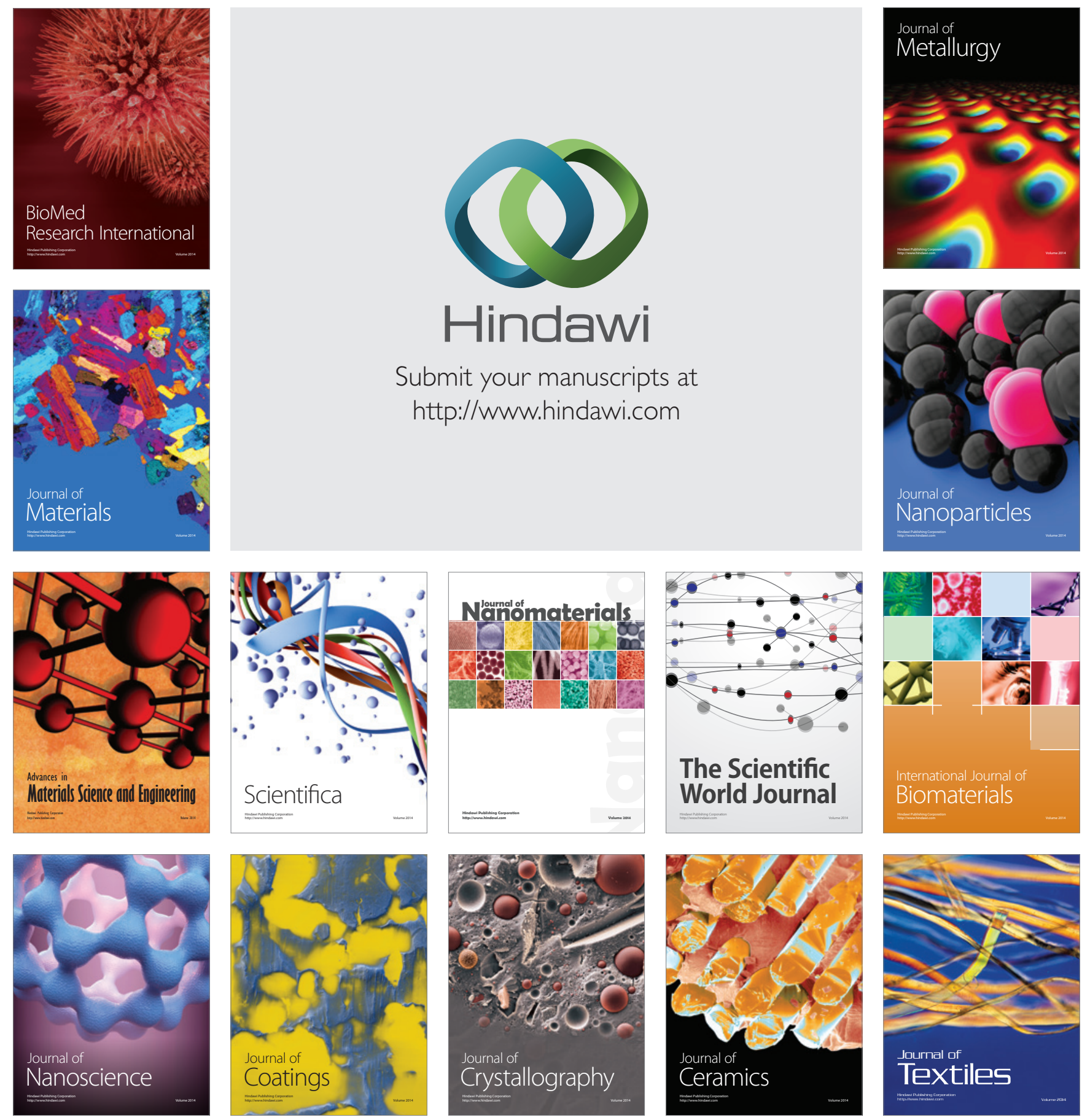